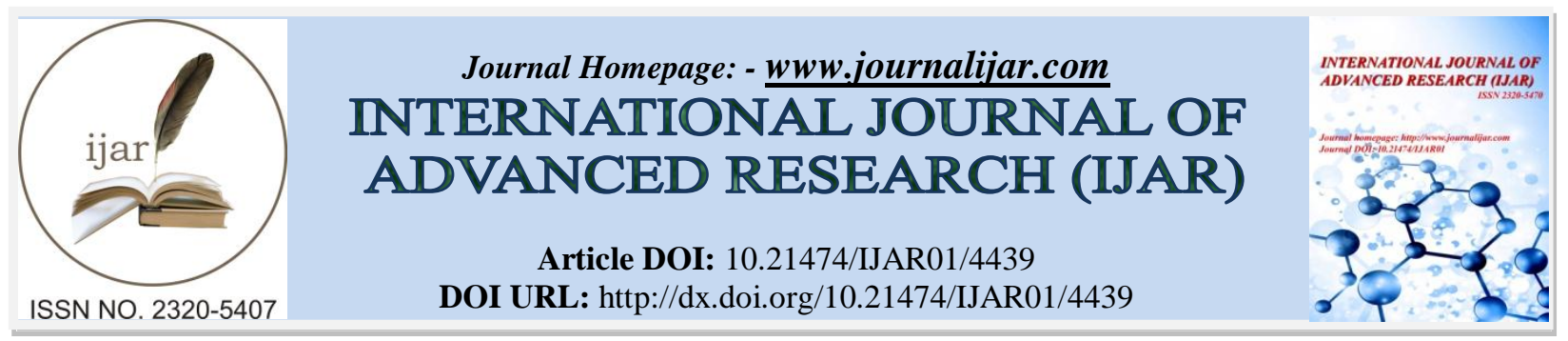

RESEARCH ARTICLE

\title{
VALIDATION OF DOPPLER FINDINGS IN BENIGN AND MALIGNANT BREAST LESION - A TERTIARY CARE EXPERIENCE.
}

\author{
Daisy Joseph V, ${ }^{1}$ Amilu Elsa Varghese ${ }^{2}$ and Sajitha $\mathbf{K}^{3}$. \\ 1. Assistant Professor, Dept of Radiodiagnosis, Govt Medical college Kottayam. \\ 2. Senior Resident ,Department of Radiodiagnosis, Govt Medical college Kottayam Kottayam. \\ 3. Additional Professor, Department of Radiodiagnosis, Govt Medical College,Kottayam.
}

\section{Manuscript Info}

Manuscript History

Received: 10 April 2017

Final Accepted: 12 May 2017

Published: June 2017

Key words:-

Breast cancer, colour Doppler, benign, Malignant

\section{Abstract}

Background:-Breast cancer is the most common malignancy in female, world wide.There is a role for screening techniques in reducing the incidence and there by morbidity of the disease. The present study aims to find out the role of Doppler findings in differentiating benign and malignant breast lesion .

Materials and methods: The study was done as diagnostic test evaluation in Department of Radiodiagnosis, who came for mammogram. Fifty patients with palpable breast lesion were included in the study. Those patients with palpable breast lesion were subjected to grey scale and colour Doppler sonography. Doppler patterns were classified into four different patterns. Results: The presence of blood flow shows high sensitivity and low specificity in differentiating benign and malignant breast lesion. The degree of vascularity is more in malignant lesion. It was shown that turbulent, high impedence flow, and venous flow were seen more in malignant lesion, when compared to benign .

Conclusion: Doppler by iteslf cannot characterize the lesions into benign and malignant lesion. When used in conjunction with conventional examination it can provide a more accurate diagnosis.

Copy Right, IJAR, 2017,. All rights reserved.

\section{Introduction:-}

Breast Cancer is the second most common Cancer worldwide with an estimated 1.68 million new cases diagnosed in 2012 representing $25 \%$ of all cancer in women ${ }^{1}$. It is estimated that by 2030 the global burden of breast cancer will increase to over 2 million new cases per year and in India it is 200000 / year . Despite an increasing incidence, mortality from breast cancer has continued to fall, thought to be the result of both earlier detection by imaging and improvements in therapy

Imaging of breast plays a crucial role in early detection of carcinoma breast which starts from screening mammogram to most modern technique of Elastography and MRI . Mammogram has a supremacy in diagnosis of lesions in a fatty breast. Exposure to ionizing radiation and difficulty in detecting lesions in dense breast are considerd as drawbacks of mammogram 
The major role of ultrasound in breast evaluation is to distinguish between cystic and solid masses and to further characterize solid masses into benign or malignant categories. In addition ultrasound is the primary modality for guided biopsies and all interventional procedures in breast disease .

Color Doppler evaluation for the presence and pattern of vascularity further helps characterization of breast masses in to benign and malignant varieties ${ }^{2}$. Tumor angiogenesis and gradient of vascularity of the mass remains the basic of color Doppler . The vascularity is important in elucidating tumor growth because a tumor cannot grow more than 1-2 mm without recruitment of new capillary blood vessels .

\section{Materials and Methods:-}

The study was designed as cross sectional study ,done in, Dept. Radiodiagnosis, Medical College Thrissur,during the period September 2010 to November 2011 . Fifty patients with a palpable breast lesion undergoing mammography were included in the study . Only patients with sonographically detectable lesions were included in further Doppler studies. Patients who didn't give consent and those with recurrences/distant metastasis were excluded from study

\section{Study Instrument:-}

Mammogram were taken using LORAD, A HOLOGIC COMPANY,M-IV and sonographic examinations were done with PHILIPS, EN VISOR HD, VERSION C .High frequency linear probe was used for the sonomammography

\section{Study Procedure and Analysis:-}

The study commenced after obtaining clearance from the Human Ethical Committee of the institution . After noting the history and clinical examination, the patients underwent mammography.

Mediolateral oblique (MLO) and Craniocaudal (CC) views were taken in all cases . Criteria For Adequate Positioning In MLO View was defined as the visulaisation of pectoralis major muscle down to the level of nipple,Inclusion of axillary tail and inframammary tissue. The CC view was considered adequate when anteroposterior distance on both sides was symmetric.

The patients were subjected to sonographic and doppler examination following mammography. The lesions were classified into benign and malignant, for each modality. Doppler examination were carried out in sonographically detected lesions .Standardized machine settings were selected to give maximum sensitivity for detection of small Doppler shifts (highest transmit power, persistence, longest velocity scale, lowest filter setting).Number of vessels were counted and each was evaluated by Doppler after adequate angle correction .If intratumoral vascularisation was detected the number of vessels were counted zero to five or greater ${ }^{2}$. Study of vessels with pulsed Doppler sonography was performed to obtained spectral wave forms for up to five different vessels. The resistive index RI\& pulsatality index PI and S/D ratio were calculated The highest RI, PI and S/D ratio obtained were considered the RI and PI and S/D ratio values for the lesions .

In this study Doppler wave forms were classified into four types ${ }^{3}$.

\section{Patterns of Doppler wave form:-}

1. Low impedance pulsatile

2. High impedance pulsatile

3. Turbulent

4. Venous

\section{Low Impedance Pulsatile Signals:-}

Low level of pulsatility and relatively high levels of diastolic flow as is seen in arteries that supply organs with low peripheral resistance.

\section{High Impedance Pulsatile Signals:-}

High impedance pulsatile signals have high pulsatility with low frequency diastolic components or no flow in diastole. 


\section{Turbulent Signals:-}

Turbulent signals were those that showed broadening of the Doppler spectrum as might be seen in a stenotic Vessels, indicating a wide range of frequencies.

\section{Venous Signals:-}

Venous flow was defined as continuous flow throughout the cardiac cycle with minimal or no variation in velocity

\section{Data Collection and Analysis:-}

Details of each patient was collected in a proforma and entered in MS Excel. All the data coded and entered in MS Excel, and analysed using SPSS 16. Tests used were proportions, chi-square tests means and independent $\mathrm{t}$ test for difference in means.

\section{Results:-}

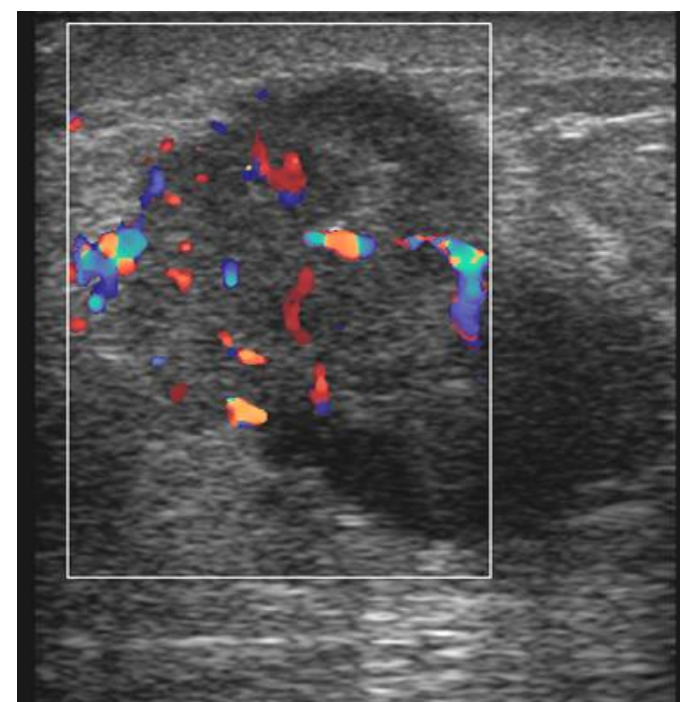

Figure1:- Colour Doppler study showing Increased number of vessels in malignant lesion.

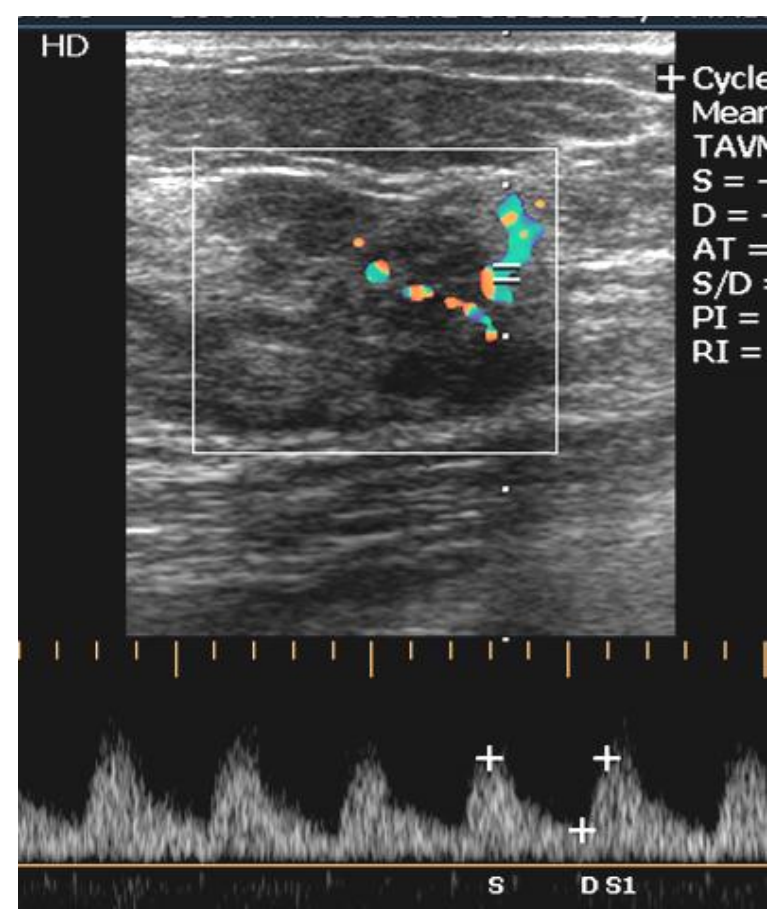

Figure 2:- Spectral pattern showing low impedance flow 


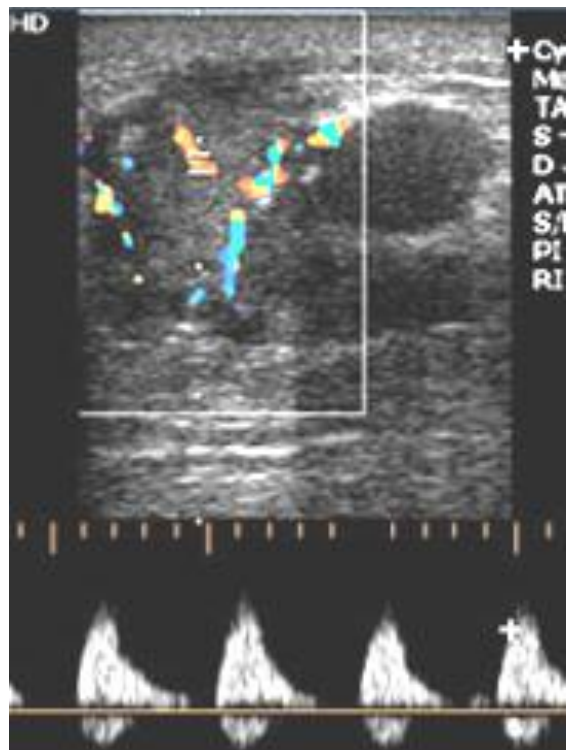

Figure 3:- Spectral pattern showing High impedance flow.

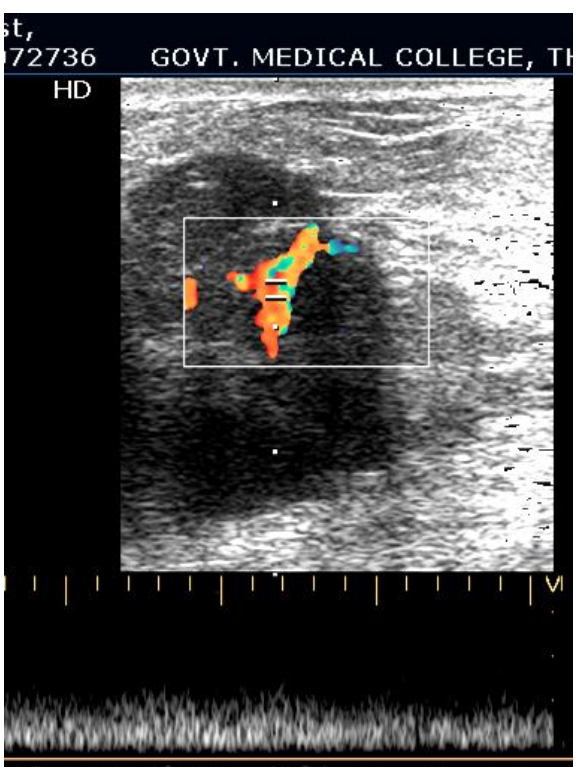

Figure 4:- Spectral pattern showing venous flow.

Table 1:- showing the Doppler parameters in benign and malignant breast lesions.

\begin{tabular}{|l|l|l|l|}
\hline Variable & Histopathological Diagnosis & P value \\
\hline & Benign(N=18) & Malignant(N=32) & \\
\hline Bloodflow & $6(33.3 \%)$ & $30(93.75 \%)$ & \\
\hline $\begin{array}{l}\text { No. of vessels }<3 \\
>3\end{array}$ & $4(66.6 \%)$ & $12(40 \%)$ & 0.230 \\
\hline Signal Type & $2(33.3 \%)$ & $18(60 \%)$ & \\
\hline Low Impedence & & & \\
\hline High Impedence & $6(100 \%)$ & $26(86.6 \%)$ & 0.002 \\
\hline Turbulent & $0(0 \%)$ & $8(26.6 \%)$ & 0.021 \\
\hline Venous & $0(0 \%)$ & $5(16.6 \%)$ & \\
\hline Resistive Index & $3(50 \%)$ & $16(53.3 \%)$ & 0.019 \\
\hline Pulsatality Index & $0.5-0.69$ & $0.6-0.89$ & \\
\hline S/D ratio & $.6-1.2$ & $1.32-1.56$ & \\
\hline
\end{tabular}


Blood Flow was demonstrated in six of eighteen benign lesions and thirty of thirty two malignant lesions giving a sensitivity of $93.7 \%$, specificity of $66.6 \%$ and positive predictive value of $83.3 \%$ for determination of malignancy based on the presence of any blood flow.

In a Significantly greater proportion of malignant lesions three or more vessels were seen . $60 \%$ of malignant lesions which showed has blood flow has more than three vessels with in the lesion compared with $33.3 \%$ of benign lesion.

However the descrimination of lesion into benign and malignant based on the number of blood vessels was wide of the mark ${ }^{4}$, as seen by a $\mathrm{p}$ value of 0.23 .

Of the lesion that showed flow, most common spectrum was of a low impedance pulsatile pattern. This was seen in $86.6 \%$ of malignant lesion and $100 \%$ of benign lesions which showed blood flow. This was not discriminatory.

However turbulent, high impedance pulsatile and venous signals were all significantly more likely to be seen in malignant lesions.

None of the benign lesion showed high impedence pulsatile or turbulent signals while $50 \%$ of those benign cases with vascularity show venous flow.

For malignant lesion ,26\% displayed high impedance pulsatile, 16\% turbulent and 53.3\% showed venous flow. Analysis showed malignant lesions significantly more likely to display more signal types of flow.

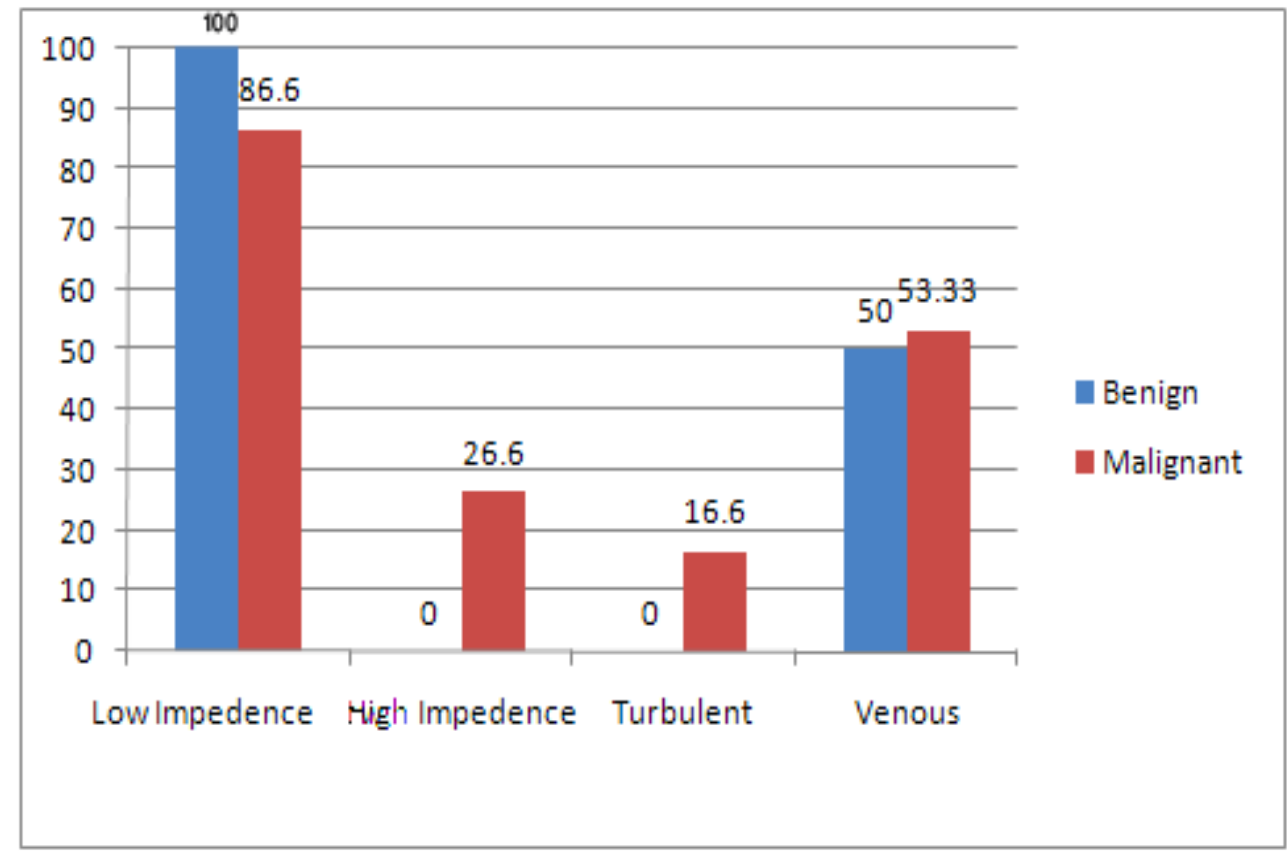

Chart 1:- showing the Signal type of Benign Vs Malignant lesions which showed colour uptake in Doppler study

It is seen from the Chart that high impedence and turbulent flow is seen only in malignant lesion

\section{Discussion:-}

Tumour angiogenesis plays an important role in the growth and extension of malignant neoplasms including those of the breast, The detection of vascularisation in the lesion is the first Doppler feature,that is used to differentiate between benign and malignant tumors .It has shown a significant association with malignancy. The logistic regression analysis in our data confirms that the malignant nature of the tumor itself is related to higher detection rates of vessels 5 . 
In this study it was able to detect vessels in $93 \%$ of breast cancers whereas only $33 \%$ of benign lesions showed vascularity. Higher percentage of detection of vascularity in malignant lesions when compared to other studies may be because only palpable lesions were included here.

In those lesions in which flow velocity curves could be obtained there was significant differences between the flow patterns for malignant and benign lesions, the former having much higher RI, PI, and S/D values than latter. This is correlating well with the study conducted by. Del Cura et $\mathrm{al}^{6}$.

The increase in resistance is related to the existence of occlusions and stenosis in the tumor vessel network structure produced by vascular encasement due to tumor growth. RI, PI and S/D values showed significant differences between benign and vascular lesions . Most of the studies done earlier showed similar higher values in malignant lesions but most of them showed overlapping with benign lesions rendering it little useful as a tool for differentiation ${ }^{7}$

For lesions that showed blood flow, consideration of pattern of flow was a helpful indicator of malignancy. The presence of high impedance and turbulent vessels and to some extent the presence of venous signals were seen mainly in malignant lesions. This shows correlating with the studies done by M.M.J McNicholas ${ }^{8}$ et al and Shen et $\mathrm{al}^{3 .}$.

\section{Conclusion:-}

In conclusion Doppler by itself is of little use when it comes to evaluating solid breast lesions.

When used in conjunction with conventional examinations it can provide a more accurate characterization of certain lesions

. The detection of vessels inside a lesion is significantly linked with neo vascularisation of malignancy.

The presence of turbulent and high impedance flow on spectral imaging is a sign although infrequent, is of high positive predictive value

Finally Histopathological conclusion is the gold standard for differentiation of tissues.

\section{References:-}

1. Ferlay J, Soerjomataram I, Ervik M, Dikshit R, Eser S, Mathers C, Rebelo M, Parkin DM, Forman D, Bray, F.GLOBOCAN 2012 v1.0, Cancer Incidence and Mortality Worldwide: IARC CancerBase No. 11 [Internet]. Lyon, France: International Agency for Research on Cancer; 2013.

2. Cosgrove DO, Bamber JC, Davey JB, Mickinna JA, Sinnet HD. Color Doppler signals from breast tumours. Radiology 1990;176:175-180

3. Shen Z-Y, Hu B, Wu M-F. Correlation between Blood Flow Signal of Color Flow Imaging and Nottingham Prognostic Index in Patients with Breast Carcinoma. Breast Care. 2012;7(2):126-130. doi:10.1159/000337766.

4. Busilacchi P, Draghi F, Preda L, Ferranti C. Has color Doppler a role in the evaluation of mammary lesions? Journal of Ultrasound. 2012;15(2):93-98. doi:10.1016/j.jus.2012.02.007.

5. Delorme S, Anton HW, Knopp MV Breast Cancer. Assessment of Vascularity by colour Doppler. Eur. Radiology 1993: 3;253-2578

6. Del Cura JL, Elizagaray E, Zabala R, Legorburu A, Grande D. The use of unenhanced Doppler sonography in the evaluation of solid breast lesions. AJR. 2005;184:1788-1794

7. The Davoudi, Yasmin et al. Role of Doppler Sonography in Distinguishing Malignant from Benign Breast Lesions Journal of Medical Ultrasound, Volume 22, Issue 2, 92 - 95)

8. Mc Nicholas MMJ, Mercer PM, Miller JC, McDermott EWN, O'Higgins NJ, McErlean DP. Color Doppler sonography in the evaluation of palpable breast masses. AJR 1993;161:765 - 771. 